Supporting Information

\title{
The Thermodynamics of Multicomponent
}

\section{Perovskites: A Guide to Highly Efficient and Stable}

\section{Solar Cell Materials}

Sungmin Kim ${ }^{1}$, Taedaehyeong Eom ${ }^{2}$, Ye-Seol Ha ${ }^{2}$, Ki-Ha Hong ${ }^{3, *}$, and Hyungjun Kim ${ }^{1, *}$

${ }^{1}$ Department of Chemistry, Korea Advanced Institute of Science and Technology, 291 Daehakro, Yuseong-gu, Daejeon 34141, Republic of Korea

${ }^{2}$ Graduate School of EEWS, Korea Advanced Institute of Science and Technology, 291 Daehakro, Yuseong-gu, Daejeon 34141, Republic of Korea

${ }^{3}$ Department of Materials Science and Engineering, Hanbat National University, 125 Dongseo-

Daero, Yuseong-Gu, Daejeon 34158, Republic of Korea 


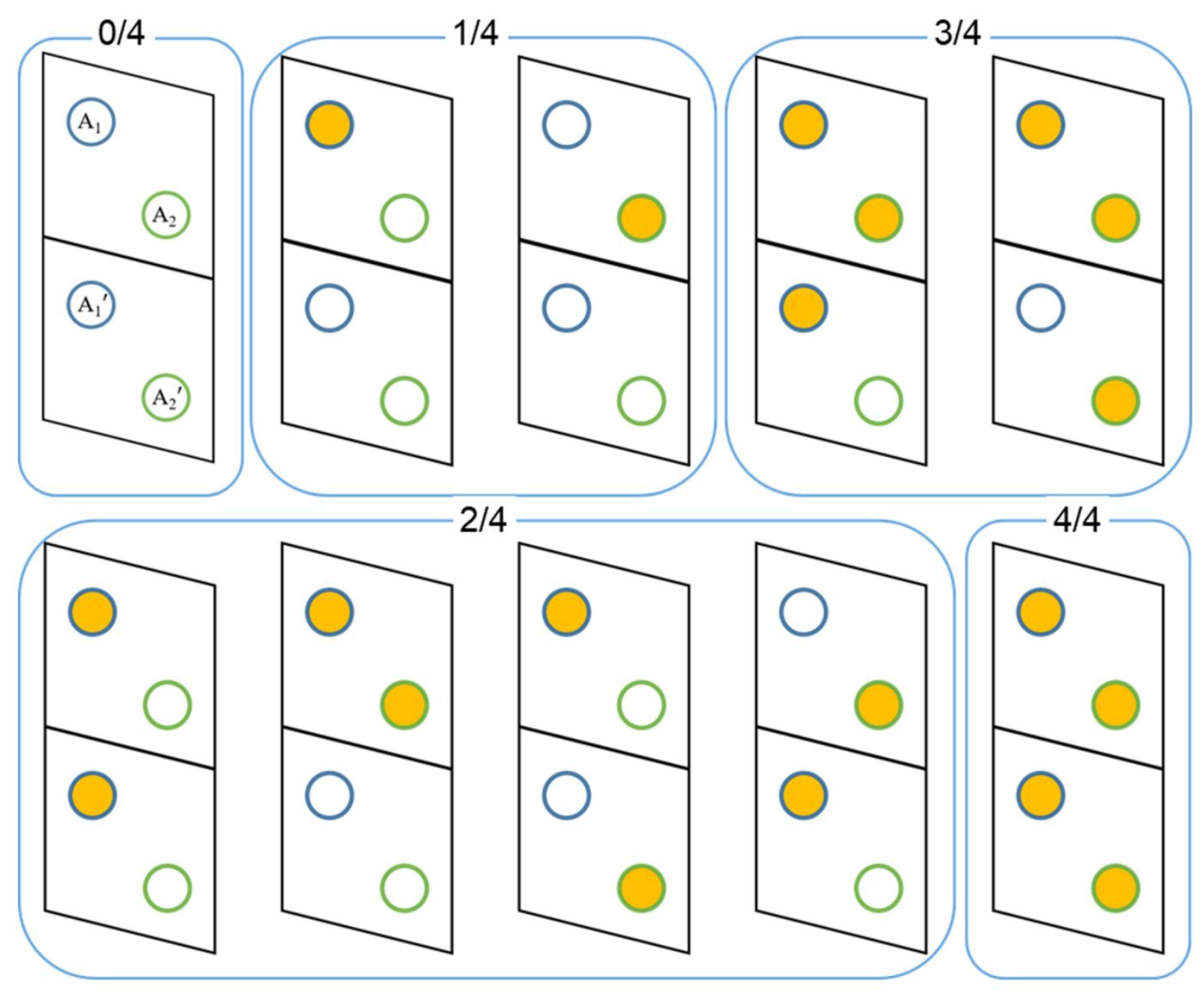

Fig. S1. 10 irreducible cation configurations in $(2 \times 1 \times 1)$ supercell of $\delta$-phase hexagonal perovskite structure. The unit cell has two different cation sites - $A_{1}$ and $A_{2}$ - in the unit cell. These sites are replicated as A1' and A2' in the supercell. After reducing configurations regarding the supercell symmetry, only 10 different case remains among 16 cases. 

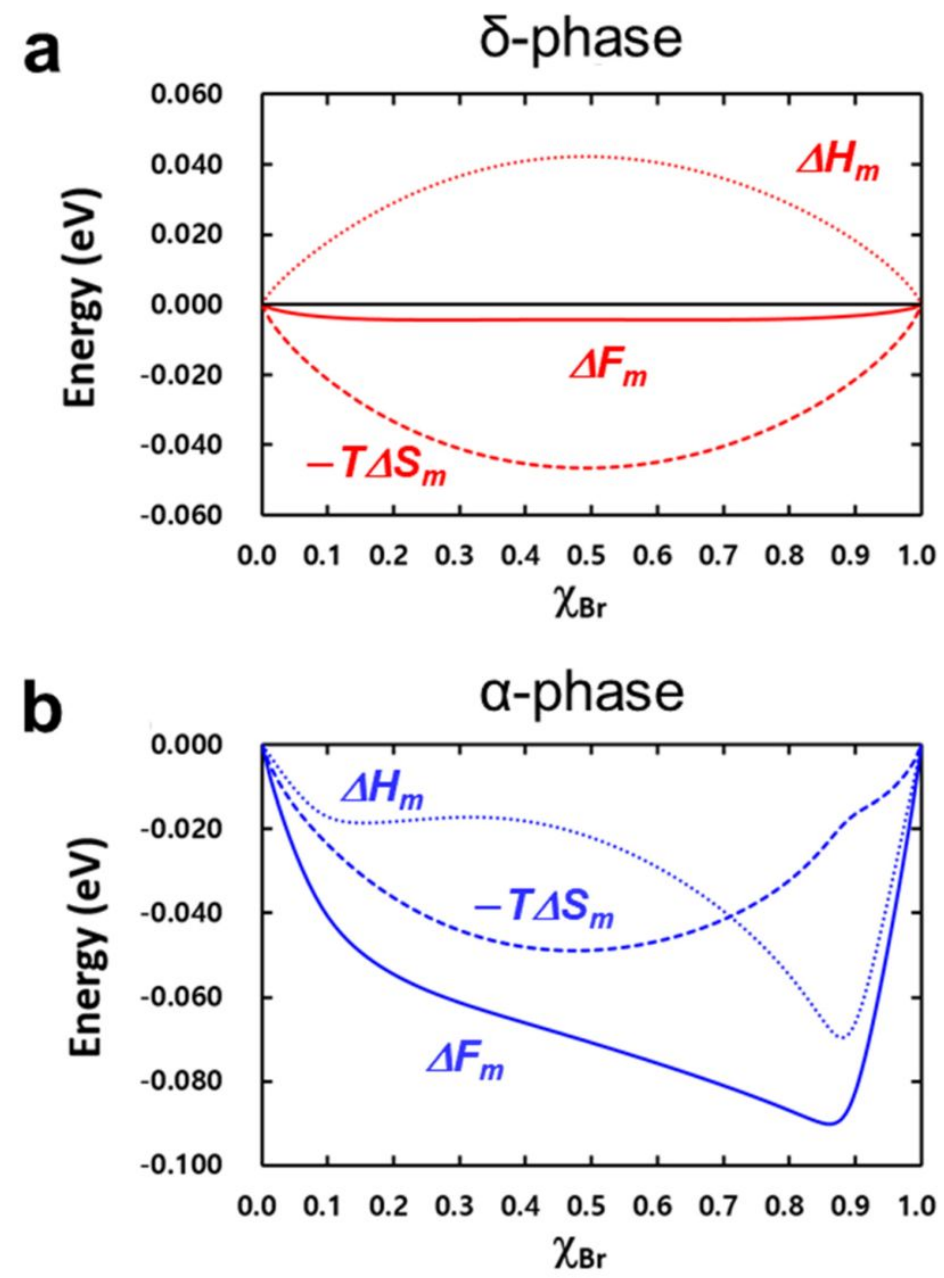

Fig. S2. Enthalpy, entropy, and free energy of binary mixing of Br and I at X-site calculated using DFT+SOD+GQCA for (a) hexagonal $\delta$-phase, and (b) trigonal $\alpha$-phase of FAPbI ${ }_{3}$. Reprinted in part with permission from ref. 28. Copyright 2017 American Chemical Society. 